REGARDS

SUR LECONOMIE ALLEMANDE

BULLETIN ECONOMIQUE DU CRAC

\section{Regards sur l'économie allemande}

Bulletin économique du CIRAC

$85 \mid 2008$

Varia

\title{
Le groupe Würth : un champion de la croissance
}

\section{Solène Hazouard}

\section{OpenEdition}

\section{Journals}

Édition électronique

URL : http://journals.openedition.org/rea/508

DOI : $10.4000 /$ rea. 508

ISBN : 978-2-8218-0866-9

ISSN : 1965-0787

Éditeur

CIRAC

Édition imprimée

Date de publication : 1 mars 2008

Pagination : 31-36

ISSN : 1156-8992

Référence électronique

Solène Hazouard, «Le groupe Würth : un champion de la croissance », Regards sur l'économie allemande [En ligne], 85 I mars 2008, document 4, mis en ligne le 01 mars 2010, consulté le 19 avril 2019. URL : http://journals.openedition.org/rea/508; DOI : 10.4000/rea.508 


\section{Le groupe Würth : un champion de la croissance}

\section{Solène Hazouard}

Leader mondial dans la vente d'éléments de fixation professionnelle, le groupe Würth est dirigé depuis le $1^{\text {er }}$ mars 2006 par la petite-fille du fondateur, Bettina Würth. Son père, Reinhold Würth, devenu une véritable référence managériale outre-Rhin, a su faire d'une petite entreprise de deux personnes un véritable géant commercial au terme de plus de cinquante années de croissance à deux chiffres. Car Würth, ce sont 8,5 milliards $€$ de chiffre d'affaires estimé pour l'année 2007, dont 5,1 milliards € à l'export, 64000 collaborateurs et un réseau de plus de 30000 vendeurs. La clé du succès, s'il en est une, est à trouver dans une approche particulière qui ne se fonde pas tant sur les techniques que sur la culture de management, cette culture reposant sur des valeurs telles que l'honnêteté, l'engagement, la modestie, la prévenance ou la réactivité, que Reinhold Würth a su transmettre à ses collaborateurs au fil du temps. Cette culture, associée à une forte orientation client soutenue par une structure spécifique où chaque entité est autonome et responsable à son niveau, représente aux yeux de ce self made man la seule chance de se différencier réellement de la concurrence, les innovations-produit ou même la qualité des produits ne permettant plus seuls de conserver un avantage compétitif stratégique.

En 1945, Adolf Würth fonde dans la ville de Künzelsau, située dans la région bade-wurtembergeoise du Hohenlohe (au nord-est de Stuttgart), une entreprise spécialisée dans le commerce de gros de vis et écrous. Il est aidé en cela par sa femme, Alma Würth qui restera membre du conseil de direction jusqu'en 1993. Lorsqu'en 1954, Adolf Würth décède à l'âge de 45 ans, c'est son fils Reinhold qui, alors à peine âgé de 19 ans, reprend la direction d'une entreprise dont le chiffre d'affaires annuel s'élève à $80000 €$.

\section{Le Hohenlohe : un cluster dans le domaine de la visserie}

En 1898, Louis et Carl Arnold fondent une première fabrique de vis dans le Hohenlohe, à Ernsbach am Kocher, qui sera rachetée près d'un siècle plus tard par Würth. Par la suite, l'industrie de la vis se développera rapidement dans la région avec la création de nombreuses entreprises dont la société Reisser, basée à Kupferzell, où Adolf Würth fera ses premières armes. En 1957, Albert Berner, ancien camarade de classe et collaborateur de Reinhold Würth, créera à son tour son propre commerce spécialisé dans la visserie. II deviendra le principal concurrent de Würth, de sorte qu'aujourd'hui, le Hohenlohe abrite en son sein non moins que le numéro 1 et le numéro 2 du commerce de vis allemand.

Le groupe Würth : chiffre d'affaires par zone géographique en 2006

\begin{tabular}{|lccr|}
\hline & CA (en millions $€$ ) & Part du CA & Salariés \\
Allemagne & 3122 & $40,3 \%$ & 15298 \\
Europe de l'Ouest & 1413 & $18,2 \%$ & 10257 \\
Europe de l'Est & 341 & $4,4 \%$ & 5225 \\
Pays scandinaves & 442 & $5,7 \%$ & 2289 \\
Europe du Sud & 1274 & $16,4 \%$ & 12054 \\
Amérique & 934 & $12,1 \%$ & 6202 \\
Asie, Afrique, Océanie & 222 & $2,9 \%$ & 3581 \\
Total & $\mathbf{7 7 4 8}$ & $\mathbf{1 0 0 \%}$ & $\mathbf{5 4 9 0 6}$ \\
\hline
\end{tabular}

Source des données : Geschäftsbericht Würth-Gruppe 2006. 
Un développement précoce à l'international

Une structure double : filiales/acquisitions

Très tôt, l'entreprise s'engage dans une politique d'internationalisation: dès 1962, à une époque où le marché allemand était encore en mesure d'offrir de larges possibilités de croissance à une PME comptant 60 employés pour un CA d'environ 2,5 millions $€$, la conquête de marchés étrangers débute avec la création de Würth Nederland B.V. aux Pays-Bas et la mise en place de filiales en Suisse et en Autriche la même année. Les autres pays européens suivront assez rapidement et en 1969, le pas de l'implantation sur le continent américain est franchi : tout d'abord aux Etats-Unis avec la société Würth Screw and Fastener Corporation, puis en Amérique du Sud avec l'établissement d'une première filiale en 1972. Deux ans auparavant, l'entreprise, forte d'un chiffre d'affaires de 32,9 millions $€$, avait déjà étendu sa présence géographique avec la création d'une filiale en Afrique du Sud. La couverture des cinq continents sera achevée rapidement avec l'implantation de Würth en Australie en 1981 puis en Malaisie en 1987, année du rachat d'une société japonaise. Depuis 1994, le groupe est également présent en Chine, de sorte qu'à l'heure actuelle, il ne compte pas moins de 400 sociétés réparties sur 86 pays.

Aujourd'hui, le groupe est structuré en deux grandes unités, chacune contribuant à hauteur de $50 \%$ à la croissance : tandis que les sous-divisions de Würth Linie sont le signe d'une adaptation de l'offre à différents segments de clientèle, les entreprises composant l'ensemble Allied Companies, issues d'un processus de rachat, répondent pour la plupart à la politique de diversification du groupe.

Le groupe Würth : chiffre d'affaires par division en 2006

\begin{tabular}{|c|c|c|c|}
\hline Divisions & $\mathbf{C A}$ (en millions $€$ ) & Part du CA & Nombre de salariés \\
\hline Würth Linie & & & Vendeurs uniquement \\
\hline Auto & 1213 & $15,7 \%$ & 9447 \\
\hline Métal & 1307 & $16,9 \%$ & 7731 \\
\hline Bois & 1034 & $13,3 \%$ & 3748 \\
\hline Construction & 439 & $5,7 \%$ & 2273 \\
\hline Industrie & 533 & $6,9 \%$ & 424 \\
\hline Allied Companies & & & Collaborateurs \\
\hline Commerce électrique de gros & 753 & $9,7 \%$ & 2305 \\
\hline Outillage & 375 & $4,8 \%$ & 1462 \\
\hline Groupe Reca & 424 & $5,5 \%$ & 3730 \\
\hline Distribution & 630 & $8,1 \%$ & 2542 \\
\hline Production & 400 & $5,2 \%$ & 3653 \\
\hline Electronique & 287 & $3,7 \%$ & 2436 \\
\hline Visserie et éléments normalisés & 202 & $2,6 \%$ & 920 \\
\hline Services financiers & - & - & 238 \\
\hline Diversification*) & - & - & - \\
\hline Total collaborateurs & 7748 & $100 \%$ & 54906 \\
\hline
\end{tabular}

Source des données: Geschäftsbericht Würth-Gruppe 2006. *) Entreprises de services de dimension régionale (hôtels, restaurants, services logistiques, Junior Entreprises...).

L'automobile : un secteur stratégique
Le développement de Würth Linie a débuté par l'automobile : en 1950, le jeune apprenti Reinhold Würth, alors âgé de 15 ans, se rend seul en déplacement clientèle auprès d'un garage Volkswagen et découvre les besoins spécifiques de ce secteur. Ce fut une expérience clé. Dès lors, il surprend par sa capacité à adapter les produits de l'entreprise pour résoudre les problèmes de ses clients, ce qui lui vaut un net avantage concurrentiel qui ne sera jamais démenti : encore aujourd'hui, l'entreprise compte les garages Volkswagen parmi ses clients.

Et l'automobile demeure au cœur de la stratégie du groupe : Würth, malgré sa position de leader, ne réalise toujours que $5 \%$ de parts de marché mondiales, les $95 \%$ restants constituant ainsi un réel potentiel que le groupe a choisi d'évaluer pays par pays selon le nombre d'automobiles en circulation, révélateur de la capacité économique d'un territoire donné. 
La division Métal de Würth Linie réalise quant à elle non moins de $17 \%$ du CA du groupe. Elle compte parmi ses clients des serruriers, des entreprises artisanales dans les domaines sanitaires et électriques/électroniques ainsi que des ateliers d'entreprises industrielles spécialisés dans la transformation du métal. Par ailleurs, la clientèle traditionnelle de la division Bois qui, pour la première fois en 2006, a dépassé le milliard d'euros de CA, rassemble des menuisiers et des fabricants de fenêtres. La division Construction approvisionne de son côté des entreprises de construction et de gros œuvre tandis que la division la plus récente, consacrée à l'Industrie livre à des entreprises de toutes tailles et de toutes branches de petits éléments de fixation utilisés dans la production.

La seconde unité du groupe, Allied Companies, a vu sa source dans l'acquisition d'un commerce de vis berlinois en 1966. Depuis lors, Würth a procédé au rachat de plus de 100 sociétés, aussi bien dans le secteur de la production que du commerce de gros. En 1980, les entreprises de commerce de gros de visserie ont été rassemblées pour constituer une structure indépendante, Reca-Norm GmbH \& Co. KG, qui a poursuivi son expansion jusqu'à s'implanter en Chine en 2004 et qui possède aujourd'hui près de 40 filiales sur le continent européen. Cette société, en concurrence directe avec la maison-mère de par son offre de produits et de services, pousse non seulement la compétitivité au sein du groupe mais vient également renforcer le pouvoir de Würth en termes de distribution, la segmentation du marché qui en découle se traduisant par la présence de deux équipes de vente distinctes. Outre cette stratégie commerciale, la nécessité d'améliorer les conditions d'approvisionnement et le souhait d'obtenir une plate-forme de développement de produits innovants ont été déterminants dans le rachat d'entreprises de production, ce qui permet également de minimiser les risques de dépendance face à un fournisseur dont la position serait dominante sur le marché.

Mais les 264 Allied Companies du groupe, réparties sur neuf divisions, sont d'abord le signe d'un processus de diversification qui a débuté avec l'établissement d'une entreprise de construction (Würth Bau) en 1970 : le plus gros échec et l'une des seules fermetures d'entreprises (en 1984) qu'ait connues le groupe au cours de son existence. En revanche, la production de cartes imprimées au sein de Würth Elektronik, lancée en 1971 mais longtemps déficitaire, est devenue un secteur rentable avec plus de 150 millions $€$ de CA et plus de 1000 employés si l'on y intègre la production de photopiles. Car Würth est aussi à la pointe de la technologie pour ce qui est de l'énergie solaire : en octobre 2006, une unité de production en série de photopiles - ou cellules photovoltaïques CIS a été inaugurée à Schwäbisch Hall sur le site de la filiale Würth Solar créée sept ans plus tôt. Le cuivre, l'indium et le sélénium (CIS) remplacent ici le silicium (Si) dans l'élaboration de semi-conducteurs de cellules photovoltaïques. Les panneaux solaires constitués de cellules CIS, dont le rendement est certes à peu près égal (12\%) à ceux composés de silicium, offrent tout de même l'avantage d'une utilisation plus flexible : il sera possible de les intégrer aux façades des bâtiments, voire à terme aux parois de tentes.

Ces dernières années, la politique de diversification est allée de pair avec la volonté d'aborder le marché externe des services financiers, dans le prolongement logique de l'activité de distribution. S'inspirant de la réussite avérée de General Electric dans ce domaine, Reinhold Würth a pu s'appuyer dans l'élaboration de cette nouvelle division sur l'expérience de ses sociétés de financement, situées en Suisse et aux Pays-Bas, en termes d'attribution de crédits en interne, de gestion des risques financiers, d'affacturage et de refinancement. Outre une entreprise de leasing et un courtier d'assurance spécialisé basé en Allemagne, cette ouverture au marché repose sur trois piliers : la société de planification budgétaire Würth Financial Services dont l'activité s'étend principalement sur le territoire de la Suisse alémanique, une participation au sein de la banque Bankhaus Bodensee AG, d'abord minoritaire en 2005, puis majoritaire (88,5\%) depuis janvier 2006 et un courtier en assurance basé en Suisse (Oberhänsli \& Partner) dont le groupe a fait l'acquisition en 2005.
Des divisions
adaptées au type de clientèle

Les Allied Companies : un soutien à la distribution et à la production...

... et une voie vers la diversification

La conquête du marché externe des services financiers 
Une croissance à deux chiffres grâce à la maîtrise d'une structure complexe
Au vu de cette structure complexe et éclatée à l'échelle globale, on peut se demander comment Würth, dont la gamme de produits comprend aujourd'hui plus de 100000 références, est devenu synonyme d'une croissance à deux chiffres (de $26 \%$ en moyenne pendant la période 1954-2004). La réponse réside certainement dans un développement de proche en proche qui, parallèlement à la politique de rachats, se fonde sur deux concepts privilégiant l'autonomie sur différents niveaux opérationnels. Le premier concept, appelé en interne «principe de la graine », souligne la démarche entrepreneuriale du groupe : les profits réalisés par la filiale sont destinés à en constituer les fonds propres, le soutien financier de la maison-mère se limitant strictement à la mise à disposition du capital de départ ainsi qu'à l'octroi de crédits à taux avantageux pour le financement de l'actif circulant; on comprend alors que le choix du futur chef d'entreprise revête une importance capitale. Selon le second concept, ou « règle du $100 \%$ », la totalité du capital social revient au groupe : lors de l'apparition d'une nouvelle filiale sur le marché, tout partenariat commercial est exclu, de sorte à conserver une pleine et entière autonomie financière.

\section{L'orientation client comme concept d'entreprise}

L'une des clés de la réussite la distribution directe

L'accent est mis sur la gestion des ressources humaines

Des procédures de vente clairement établies

La qualité du service client
Bien que le groupe, fortement orienté vers la distribution et ne produisant en propre que $5 \%$ de son CA, ait vu sa gamme de produits s'élargir considérablement depuis 1945, son modèle économique de vente directe n'a guère évolué en soixante années d'activité. Le succès de l'entreprise est ainsi à attribuer certes moins à l'innovation-produit (les éléments de fixation demeurent très classiques et la plupart d'entre eux, normalisés DIN, ne diffèrent guère des produits de la concurrence) qu'à l'idée de distribution directe, dans les locaux mêmes du client, qui, lorsqu'elle fut énoncée par Reinhold Würth dans les années 1950, était en rupture totale avec les règles établies par les grossistes en visserie traditionnels.

Pour rester au plus près du client (en 2007, les 30600 vendeurs de Würth suivaient plus de 2,9 millions de clients répartis sur 86 pays), l'accent est mis sur la gestion des ressources humaines, notamment auprès des vendeurs. La performance de tout vendeur - ou de toute filiale - est contrôlée chaque mois de manière rigoureuse via le système d'information du groupe (Würth-Info-System, WIS). C'est cette même performance qui détermine non seulement les avantages accordés aux vendeurs (la marque et le modèle de la voiture de fonction dépendent directement du résultat atteint, de sorte que le client sait dès le premier coup d'œil s'il a affaire à un bon vendeur ou non) mais aussi et surtout leur niveau de rémunération, la part fixe ne constituant qu'une petite partie du salaire final. On peut alors comprendre que certains vendeurs choisissent de quitter l'entreprise, le turnover annuel atteignant les $9 \%$.

Dans le cadre de la procédure «action et information de vente » (Verkaufsinformation- und aktion, VIA), chaque vendeur doit suivre sur une base annuelle un « mini business plan » dans lequel un objectif en termes de CA est fixé selon le temps de travail disponible (les ressources) et le nombre de clients présents sur le secteur géographique donné (le potentiel). Un business plan-type contient des objectifs tels l'acquisition de nouveaux clients, la reconquête d'anciens clients, le placement de nouveaux produits, la rapidité à traiter les réclamations ou la réduction du nombre de réclamations, une structure de clientèle équilibrée, la visite régulière des clients, une vente adaptée aux besoins et la résolution des problèmes rencontrés par le client, ou encore l'application des systèmes disponibles pour la gestion du secteur, l'aide apportée par les collègues et l'analyse régulière du secteur en vue d'un bilan de compétences, cet instrument ayant pour principale visée de développer l'offre de produits ou de services par l'analyse de potentiels encore inexploités.

Outil supplémentaire d'orientation client : l'Académie Würth, porteuse d'une offre de formation continue, par l'intermédiaire de laquelle près de 7000 clients ont pu participer en 2006 à des séminaires dans leurs propres locaux ou au sein des 54 
centres d'artisans que compte le groupe. Par ailleurs, le contact avec le client est stimulé par l'obligation pour chaque cadre dirigeant d'accompagner un vendeur sur le terrain au moins quatre fois par an: Reinhold Würth donne lui-même l'exemple en consacrant chaque année deux à trois semaines à des déplacements clientèle. En parallèle, en interne, le nombre de sonneries comptabilisées lors d'un appel téléphonique avant que l'interlocuteur ne décroche est régulièrement contrôlé, et ce, en vue d'améliorer constamment la qualité du service client, garanti également par le fort degré d'autonomie des différentes filiales et centres de profits du groupe, responsables de leurs propres pertes et bénéfices.

\section{Un management adapté à une structure globale complexe}

Dans le négoce de procédés de fixation destinés à la production, Würth dispose d'une position stratégique à l'interface entre le producteur et le client, qui d'un côté, procure au département achats un certain pouvoir sur les fabricants du Mittelstand et, de l'autre, limite les risques de dépendance envers quelques gros clients. La concurrence est constituée essentiellement de nombreuses PME locales, le plus gros concurrent sur le marché mondial étant l'entreprise Berner qui n'atteint cependant pas les 700 millions $€$ de chiffre d'affaires malgré une bonne rentabilité, une croissance de CA continue et un modèle économique similaire à celui de Würth.

Le succès du groupe repose alors en grande partie sur la personnalité de celui qui a su élever un petit commerce local au rang mondial. Nommé docteur honoris causa en économie d'entreprise à l'université de Tübingen et Professeur de management à la Technische Hochschule de Karlsruhe, Reinhold Würth a su faire profiter l'entreprise de sa compréhension des enjeux managériaux (au-delà de toute mode de management ou de toute simplification réductrice) et de sa capacité à focaliser constamment l'attention de l'organisation dans son ensemble sur des facteurs essentiels de réussite. Ainsi, c'est par l'intuition qu'ont été développés les instruments et concepts de management actuellement en vigueur au sein du groupe qui ont aujourd'hui valeur de modèle pour nombre de PME familiales, ce modèle étant devenu un véritable cas d'école en management (Venohr, 2006). II n'y a alors rien de surprenant à ce que se tienne en mai 2008 un séminaire dans le cadre duquel un ancien collaborateur de Würth, Dieter Krämer, présentera les facteurs de réussite de l'entreprise.

La structure de Würth, qu'on peut considérer a posteriori comme fractale, repose sur l'autonomie et la responsabilité de chacun et exige par là même une forte réactivité à tous les niveaux, un retour permanent de l'information. Quand on sait qu'une commande moyenne ne dépasse pas les $220 €$, la gestion des stocks se doit d'être irréprochable : elle est soutenue par le système breveté ORSY, grâce auquel les clients ont la possibilité d'entreposer et d'extraire de petits éléments de fixation, également appelés produits 'C'. Ceci, prolongé par une logique d'approvisionnement Kanban relayée par l'entreprise Würth Industrie Service $\mathrm{GmbH}$ \& Co. KG., offre aux clients industriels des possibilités de rationalisation de la chaîne logistique. Par ailleurs, la gestion de l'approvisionnement d'entreprises multinationales ayant des sites de production dans plusieurs pays est assurée via le réseau d'entreprises Würth Industrial Network (WINWork), qui propose ainsi à ses clients un service international et coordonné.

L'amélioration continue, appelée Kaizen chez Toyota, constitue en outre une approche managériale valorisée depuis de nombreuses années chez Würth dont la logique d'excellence qualitative déteint sur ses fournisseurs, ces derniers devant se soumettre à des contrôles permanents réalisés par les chefs de produit.

Le positionnement de l'entreprise en leader incontesté sur son marché étant l'œuvre de toute une vie, la question de la succession, qui s'est déroulée en plusieurs étapes, en devient d'autant plus délicate : en 1984 et 1987, Reinhold Würth crée cinq fondations familiales (au nom de son père, de sa femme Carmen
Une concurrence éclatée, composée principalement de PME

Un succès né de l'intuition

L'importance du facteur logistique...

... et de l'amélioration continue

Reinhold Würth / Bettina Würth : une succession achevée 
et de ses trois enfants Marion, Bettina et Markus) qui détiennent la totalité du capital réparti sur différents holdings regroupant l'ensemble des sociétés du groupe. Puis, en 1994, il renonce à la direction opérationnelle et devient président du conseil de surveillance pour nommer en 2006 sa fille Bettina à la tête de cette instance, conservant tout de même une certaine marge de manœuvre dans sa nouvelle fonction de président d'honneur. Le pouvoir économique reste dès lors entre les mains de la famille Würth, de même que le pouvoir décisionnel et stratégique.

Bettina Würth a repris les rênes du groupe après cinq années d'activité au sein du comité de direction opérationnelle. Fidèle à la culture d'implication active dans le développement régional qui caractérise le patronat allemand (voir $R E A 84 / 07$ ), elle est également vice-présidente de la Chambre de commerce et d'industrie de Heilbronn-Franconie. Dotée elle aussi d'une personnalité forte dont atteste un parcours atypique (n'ayant jamais passé le bac, elle a d'abord travaillé comme éducatrice de jeunes enfants pour commencer à 23 ans une formation d'agent technico-commercial au sein de l'entreprise familiale), Bettina Würth a fondé en 2006 l'École Libre Anne-Sophie visant à développer les compétences sociales des plus jeunes, portée financièrement par la fondation d'utilité publique Stiftung Würth qui depuis de nombreuses années soutient les activités de mécénat de l'entreprise (voir le site www.stiftung-wuerth.de).

LE SECRET DE LA RÉUSSITE DE WüRTH tient essentiellement à la personnalité de l'un de ces managers typiques des années de reconstruction de l'économie allemande qui, s'appuyant sur des valeurs fortes issues d'une culture d'artisan, a fait progressivement de sa société un modèle à suivre pour de nombreuses entreprises familiales. Mais c'est également la raison pour laquelle ce modèle, bien qu'éprouvé par plus d'un demi-siècle de croissance à deux chiffres, n'est pas nécessairement applicable à d'autres structures. En effet, "la croyance en des solutions standard est une illusion. La reprise dépourvue de sens critique de 'clés du succès' bien définies mène souvent à des effets nuls, voire dans le pire des cas à des effets négatifs" (Venohr, 2006). Toutefois, au sein du groupe, le partage des valeurs d'une génération à l'autre laisse à penser que la culture d'entreprise, qui a grandement contribué au succès de Würth, perdurera ces prochaines années.

\section{Indications bibliographiques}

BouRgeols I. (dir.), Allemagne : compétitivité et dynamiques territoriales, Coll. Travaux et Documents du CIRAC, Cergy-Pontoise, juillet 2007

BOURGEOIS I., "Entreprises familiales : un rôle clef outre-Rhin », Regards sur l'Economie Allemande, $\mathrm{n}^{\circ} 82 / 2007$

GuIOT C., "Wacker Chemie AG : une familiale cotée en bourse », Regards sur l'Economie Allemande, $n^{\circ} 83 / 2007$

KLEMPKENS W., " Fotovoltaik - Eine neue Generation von Solarzellen liefert mehr Strom zu deutlich niedrigeren Kosten », Wirtschaftswoche, n 43, 23.10.2006

Venohr B., Wachsen wie Würth - Das Geheimnis des Welterfolgs, Campus Verlag, Francfort, 2006

WeISSMAN A., Die großen Strategien für den Mittelstand - die erfolgreichsten Unternehmer verraten ihre Rezepte, Campus Verlag, Frankfurt, 2006

WiLdhagen A., " Nicht nur Töchterle », Handelsblatt, 6. Oktober 2006

ZIESEMER B., Pioniere der deutschen Wirtschaft - Was wir von den großen Unternehmerpersönlichkeiten lernen können, Campus Verlag, Frankfurt, 2006 www.wuerth.de, www.wuerth.com. 